\title{
Efecto parasítico de Trichogramma y del depredador Chrysoperla sobre huevos de Compsus viridivittatus, plaga de Vitis vinifera, en laboratorio
}

\section{Parasitism of Trichogramma and predation of Chrysoperla on Compsus viridivittatus eggs, a Vitis vinifera pest, under laboratory conditions}

DIEGO ALONSO PINZÓN-HAMÓN' 1 JOHN WILSON MARTÍNEZ-OSORIO',3

MAYERLY ALEJANDRA CASTRO-LÓPEZ²

Individuos del picudo, C. viridivittatus, confinados en condiciones de laboratorio, UPTC, Tunja (Colombia).

Foto: D.A. Pinzón-Hamón

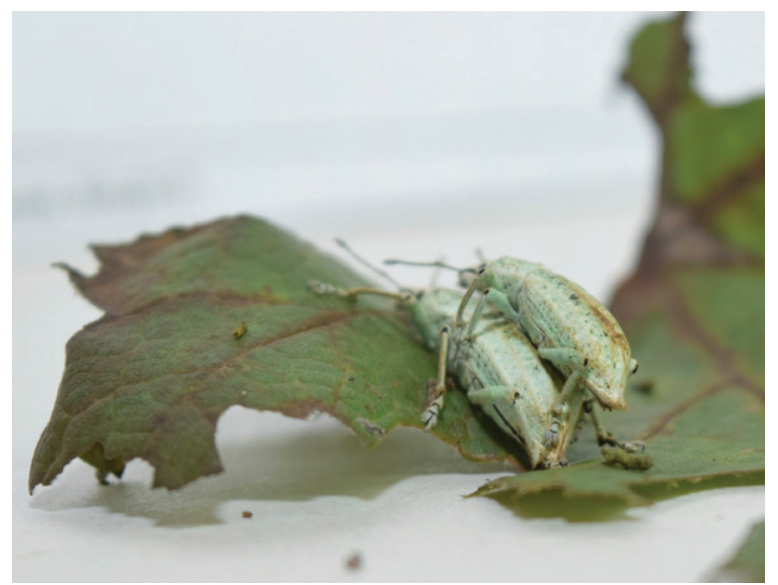

\section{RESUMEN}

Se evaluaron los potenciales parasíticos de Trichogramma exiguum y Trichogramma pretiosum y del depredador Chrysoperla carnea sobre huevos del picudo Compsus viridivittatus, plaga presente en cultivos de vid en Boyacá, Colombia. En ensayos con T. exiguum y T. pretiosum, se emplearon grupos de 240 huevos del picudo expuestos a densidades de 0, 15, 30 y 45 parasitoides. En el ensayo con C. carnea se empleó una larva de segundo instar del depredador y densidades de presa de 80, 160 y 240 huevos de C. viridivittatus. Los ensayos se establecieron en condiciones de laboratorio $\left(18 \pm 2{ }^{\circ} \mathrm{C}\right.$ y $\left.60 \pm 5 \% \mathrm{HR}\right)$ con un diseño experimental completamente al azar. En los ensayos con T. exiguum y T. pretiosum el mayor porcentaje de parasitismo se obtuvo con una densidad de 45 parasitoides $(69,4$ y 82,5\%, respectivamente). Se estableció que T. pretiosum fue más efectivo, parasitando huevos de C. viridivittatus que T. exiguum, originando una emergencia de larvas inferior al $13 \%$ y parasitismo superior al 70\%. El ensayo con C. carnea evidenció que el mayor nivel de consumo (85,8\%) se obtuvo con una cantidad de presas de 80 huevos de C. viridivittatus. No se presentó emergencia de los parasitoides tanto en los huevos expuestos a $T$. exiguum como $T$. pretiosum, atribuible a las condiciones ambientales y/o características intrínsecas del huésped C. viridivittatus. Los entomófagos evaluados originaron porcentajes de mortalidad en huevos del picudo, superiores al 50\%, sugiriendo su posterior evaluación en campo para establecer un posible uso en programas de control biológico de C. viridivittatus en vid en Boyacá.

Comité de Cafeteros de Santander, Bucaramanga (Colombia). ORCID Pinzón-Hamón, D.A.: 0000-0002-3676-7399

2 Grupo Manejo Biológico de Cultivos, Universidad Pedagógica y Tecnológica de Colombia (UPTC), Tunja (Colombia). ORCID Martínez-Osorio, J.W.: 0000-0002-5742-8062; ORCID Castro-López, M.A.: 0000-0003-1151-2514

3 Autor para correspondencia. john.martinez@uptc.edu.co 
Palabras clave adicionales: control biológico, depredación, parasitismo, vid.

\section{ABSTRACT}

The parasitic potential of Trichogramma exiguum and Trichogramma pretiosum and the predatory effect of Chrysoperla carnea were evaluated on Compsus viridivittatus eggs, an insect pest in grape fields in Boyaca, Colombia. The T. exiguum and T. pretiosum tests used 240 eggs groups exposed to $0,15,30$ and 45 parasitoids densities. In the C. carnea test, a second instar larva was placed with prey densities of 80, 160 and 240 C. viridivittatus eggs. A completely randomized design was used under laboratory conditions $\left(18 \pm 2^{\circ} \mathrm{C}\right.$ and $\left.60 \pm 5 \% \mathrm{RH}\right)$. In the trials with $T$. exiguum and T. pretiosum, the highest percentages of parasitism were obtained with the 45 parasitoid density $(69.4 \%$ and $82.5 \%$, respectively). It was established that T. pretiosum was more effective in the parasitism of C. viridivittatus than T. exiguum, resulting in larvae emergence under $13 \%$ and parasitism over $70 \%$. The test with C. carnea showed that the highest consumption percentage (85.8\%) was obtained with the 80 C. viridivittatus egg density. There was no emergence of parasitoids from the eggs parasitized by T. exiguum and T. pretiosum, probably because of the environmental conditions and/or C. viridivittatus host intrinsic characteristics. The tested entomophagous species showed over $50 \%$ C. viridivittatus egg mortality, suggesting that they can be used in future field tests in grape vine crops in order to observe their potential in C. viridivittatus biological control programs in Boyaca.

Additional key words: biological control, parasitism, predation, grape.

Fecha de recepción: 02-03-2018 Aprobado para publicación: 30-05-2018

INTRODUCCIÓN

En Colombia el cultivo de uva está presente en varias regiones y en el departamento de Boyacá se ubica en zonas de clima frío localizadas en las provincias del alto Ricaurte, Sugamuxi, Tundama, Valderrama y Norte, en producciones comerciales exclusivas para la elaboración de vinos (Almanza et al., 2012).

En la actualidad, uno de los factores limitantes en la producción de uva, es el picudo C. viridivittatus (Coleóptera: Curculionidae), insecto de aparición reciente en el cultivo de vid en Boyacá, el cual según observaciones en campo, causa defoliación con la consecuente pérdida de vigor de las plantas y reducción de la producción de uva y deterioro de su calidad.

El potencial reproductivo de este insecto es alto; la hembra puede colocar posturas compuestas desde 53 a 94 huevos cada una, con más de 3.500 huevos ovipositados durante su etapa adulta. El ciclo de vida puede durar entre 134 a 268 d, con 1,36 a 2,72 generaciones por año, en las cuales el insecto adulto consume follaje, realizando cortes irregulares en los bordes de las hojas y las larvas que son rizófagas, consumen la corteza de la raíz (Cano et al., 2002).

En evaluaciones realizadas en cultivos de vid se ha determinado la presencia del picudo de los cítricos $C$. viridivittatus en poblaciones que sobrepasan el umbral de daño económico (Rincón et al., 2015). Este es un insecto polífago, que se alimenta de una gran variedad de cítricos (lima rangpur, mandarina cleopatra, naranja valenciana, tangelo mineola, toronja), ciperáceas, mataratón, palma areca, mangostino, banano, guayaba, aguacate, mango, plátano, yuca, guácimo, bejuco trifoliado, fríjol, café, algodón, maní, sorgo, maracuyá, entre otros (Peñaloza y Diaz, 2004).

El control biológico busca disminuir la población de organismos plaga, ya sea de forma temporal o permanente (Van Driesche et al., 2007) empleando diversos agentes como entomófagos (parasitoides y depredadores) o entomopatógenos. Hasta ahora, no se reporta la implementación de enemigos naturales para $C$. viridivittatus en vid como estrategia generalizada, sin 
embargo, en algunas zonas productoras de cítricos en Colombia se han documentado parasitoides como Aprostocerus sp. (Hymenoptera: Eulophidae) y Trigonospila sp. (Diptera: Tachinidae) y se ha evaluado el parasitoide Fidiobia sp. (Hymenoptera: Platygastridae) en campo, este último, exhibiendo promedios de parasitismo cercanos al 50\% en huevos de Compsus sp. (Estrada y Soto, 2011). En entomopatógenos, se observó en laboratorio el efecto de Beauveria bassiana y Metarhizium anisopliae sobre adultos del picudo, encontrándose algunos aislamientos promisorios (Marín y Bustillo, 2008; Rincón y Montoya, 2015) y aislamientos de Beauveria bassiana y Metarhizium anisoplia y nematodos Steinernema sp. y Heterorhabditis sp., obtenidos de suelos citrícolas, causaron mortalidades en adultos de C. viridivittatus entre el 94 y $100 \%$ para los hongos y 42 y $65 \%$ para los nematodos (Zuluaga et al., 2015). Se evidencia así, que el control biológico de $C$. viridivittatus puede ser una alternativa viable dentro de programas de manejo integrado de plagas en cultivos de uva.

Los parasitoides del género Trichogramma, tienen gran importancia, por incluir especies de interés en el control biológico de plagas y por su facilidad para reproducirse masivamente en laboratorio (Navarro, 1990; Oliveira et al. 2000); dentro de las especies más importantes para el control de plagas se encuentran Trichogramma pretiosum Riley y Trichogramma exiguum (García et al., 2011) como parasitoides de huevos de lepidópteros. En Coleóptera son escasos los reportes de parasitoidismo de huevos por especies de Trichogramma; sin embargo, existen estudios de Ceratogramma etienney en huevos de Diaprepes abreviatus (coleoptera: curculionidae) (Amalin et al., 2005), Haeckeliania sperata igualmente en huevos de D. abreviatus (Carrillo et al., 2008) y Uscana lariophaga en huevos de Callosobrucbus maculatus (Fabricius) y Bruchidius atrolineatus Pic, (Coleoptera: Bruchidae) (Van Huis et al., 1991). En el caso específico del género Compsus, Estrada y Soto (2011) mencionan el parasitismo de Haeckeliana sp. (Hymenoptera: Trichogrammatidae).

En cuanto a otro tipo de entomófagos, los hábitos depredadores son característicos del orden Neuróptera, entre los cuales se destaca Chrysoperla carnea (Stephens), por ser un buen controlador natural debido a su distribución geográfica, su tolerancia a insecticidas como abamectinas (Cerna et al., 2012), alta voracidad, capacidad de búsqueda y facilidad de producción en laboratorio (Nasreen et al., 2005). C. carnea es depredador de una amplia gama de artrópodos de cuerpo blando (áfidos, escamas, thrips, ácaros, etc) y estados inmaduros como huevos y larvas (Yadav y Pathak, 2010), sin embargo, no se han reportado como depredadores de C. viridivittatus, desconociéndose el potencial controlador que este grupo pudiera tener sobre el picudo. Los depredadores de Compsus sp. reportados en campo incluyen a varias especies del orden Hymenoptera, familia Formicidae, como Brachymyrme sp., Pheidole sp., Solenopsis sp. y Camponotus sp. (Estrada y Soto, 2011).

Dada la importancia económica del cultivo de vid en algunas zonas de Boyacá, y el impacto nocivo que actualmente C. viridivittatus está ocasionando en el mismo, el objetivo de esta investigación fue determinar el efecto depredador de C. carnea y el efecto parasítico de T. pretiosum y T. exiguum sobre huevos de C. viridivittatus en condiciones de laboratorio.

\section{MATERIALES Y MÉTODOS}

La investigación se realizó a partir de la colecta de individuos adultos de C. viridivittatus, en un viñedo ubicado en el Valle de Sáchica (Boyacá) (5³6’3,23" N y 7333'11,84" W). Este municipio tiene una extensión de $62,4 \mathrm{~km}^{2}$, temperatura promedio de $16,2^{\circ} \mathrm{C}$ y una altitud de $2.150 \mathrm{~m}$. El material de vid presente corresponde a la cepa Cabernet Sauvignon, proveniente en su mayor parte de Francia, y con una extensión de 6 ha. Los individuos del picudo colectados fueron trasladados posteriormente al Laboratorio de Control Biológico $\left(18 \pm 2^{\circ} \mathrm{C}\right.$ y $60 \pm 5 \%$ HR) de la Universidad Pedagógica y Tecnológica de Colombia, en la ciudad de Tunja en donde se desarrollaron las pruebas biológicas (Fig. 1A).

La corroboración taxonómica de la especie C. viridivittatus (Guérin-Méneville) se realizó empleando la clave desarrollada por O'Brien y Peña (2012), para adultos de Compsus Schoenherr (Coleoptera: Curculionidae: Entiminae) colectados en Colombia. Se identificaron los caracteres morfológicos específicos de C. viridivittatus descritos en la clave, como la extensión de una franja triangular desde el protórax hasta la base de la cabeza y la extensión de las cuatro franjas longitudinales de coloración verde con escamas metálicas sobre los élitros, entre otros.

Los parasitoides T. exiguum y T. pretiosum fueron suministrados por la empresa Bioagro Cartago SAS (Cartago, Valle del Cauca, Colombia) y el depredador Chrysoperla carnea por la empresa Perkins Ltda. (Palmira, Valle del Cauca, Colombia). 


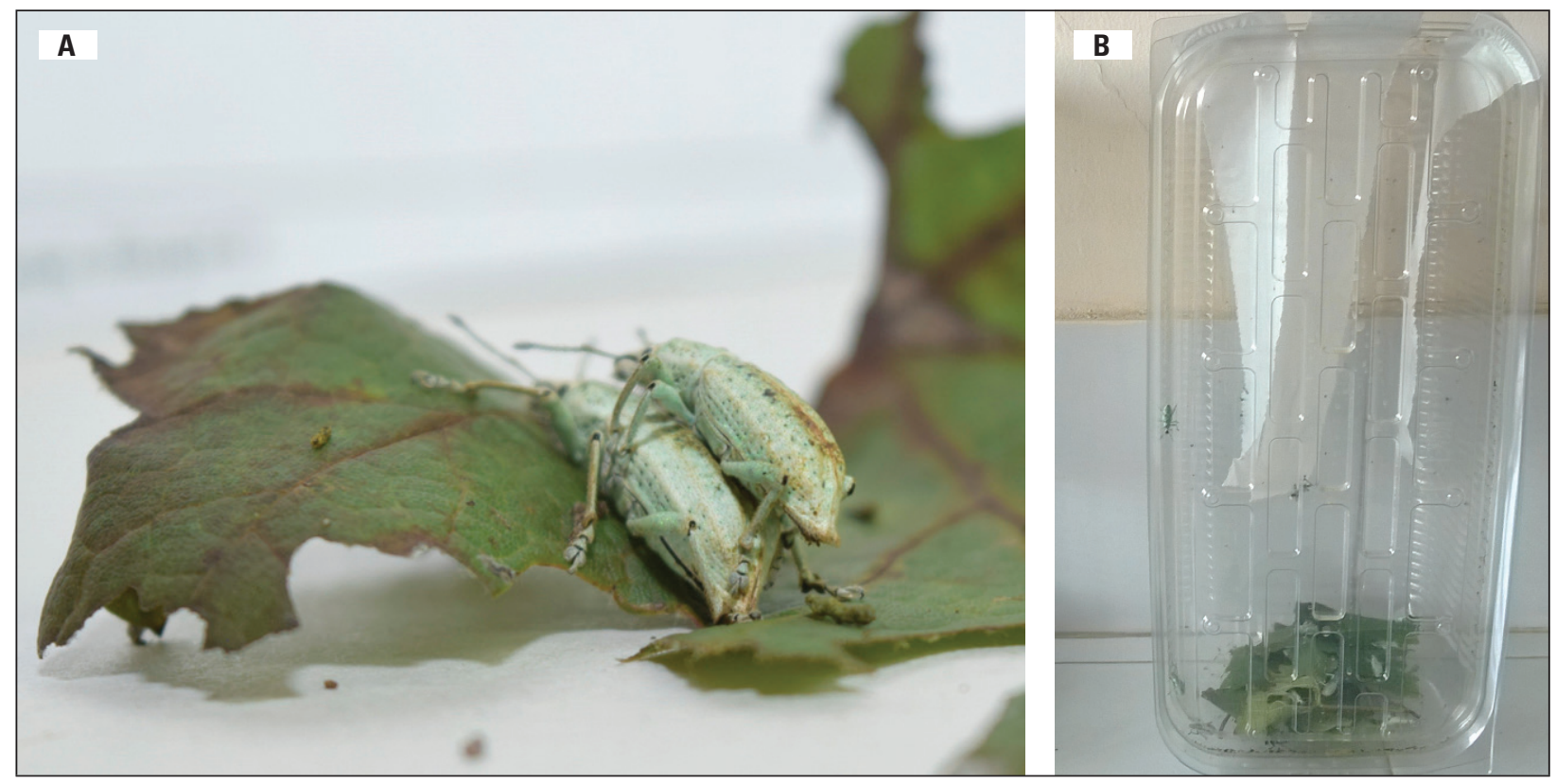

Figura 1. Individuos de C. viridivittatus, confinados en condiciones de laboratorio. A. Macho y hembra; B. Montaje de recipientes para colecta de huevos.

Los huevos de C. viridivittatus se obtuvieron de los adultos colectados en campo y mantenidos en el laboratorio. Para tal fin, se ubicaron machos y hembras en proporción 1:5, en recipientes de polietileno transparentes, de aproximadamente $2 \mathrm{~kg}$ de capacidad. Cada recipiente contenía hojas de vid como sustrato alimenticio y dos tiras dobles de plástico de $4 \mathrm{~cm}$ de ancho y $20 \mathrm{~cm}$ de largo, de color blanco, para proveer un sitio de oviposición, adaptando la metodología propuesta por Estrada y Soto (2011) (Fig. 1B).

Diariamente se revisaron las tiras plásticas y las hojas de vid, con el objeto de retirar los huevos que serían empleados posteriormente en las pruebas de parasitismo y depredación.

\section{Potencial parasítico de T. pretiosum y T. exiguum en huevos de $C$. viridivittatus}

En la evaluación de los parasitoides se emplearon grupos de 240 huevos de C. viridivittatus, presentes en las tiras de plástico blanco antes mencionadas, evitando su manipulación, para no afectar su desarrollo normal. La unidad experimental consistió en envases de plástico transparentes, con una capacidad de 32 onzas, con ventilación, pero cierre hermético, en donde se ubicaron los huevos. En cada envase se introdujeron posteriormente los individuos del parasitoide a evaluar (T. pretiosum o T. exiguum) empleando la presentación comercial de los mismos y ajustada según lo requerido. Los seis tratamientos evaluados se describen en la tabla $1 \mathrm{~A}$.

Tabla 1. Tratamientos experimentales empleados en laboratorio en las pruebas de parasitismo (A) y depredación con huevos (B) de $C$. viridivittatus.

\begin{tabular}{|c|c|c|c|}
\hline \multicolumn{4}{|l|}{$A$} \\
\hline Ensayo & Tratamiento & $\begin{array}{l}\text { Especie de } \\
\text { parasitoide }\end{array}$ & $\begin{array}{c}\text { Parasitoides/unidad } \\
\text { experimental }\end{array}$ \\
\hline \multirow{3}{*}{1} & $\mathrm{~T} 1$ & T. pretiosum & 15 \\
\hline & $\mathrm{T} 2$ & T. pretiosum & 30 \\
\hline & T3 & T. pretiosum & 45 \\
\hline \multirow{4}{*}{2} & $\mathrm{T1}$ & T. exiguum & 15 \\
\hline & $\mathrm{T} 2$ & T. exiguum & 30 \\
\hline & T3 & T. exiguum & 45 \\
\hline & Testigo & - & 0 \\
\hline \multicolumn{4}{|l|}{$B$} \\
\hline Ensayo & Tratamiento & $\begin{array}{l}\text { Larvas de } \\
\text { depredador/ } \\
\text { recipiente }\end{array}$ & $\begin{array}{l}\text { Huevos/unidad } \\
\text { experimental }\end{array}$ \\
\hline \multirow{4}{*}{3} & $\mathrm{~T} 1$ & 1 & 80 \\
\hline & T2 & 1 & 160 \\
\hline & T3 & 1 & 240 \\
\hline & Testigo & 0 & 240 \\
\hline
\end{tabular}


Transcurridas $72 \mathrm{~h}$ se retiraron los parasitoides del recipiente y se continuó con el seguimiento al desarrollo de los huevos del picudo. Las variables evaluadas diariamente fueron: número de larvas de C. viridivittatus emergidas, número de huevos parasitados y número de individuos emergidos de cada especie de parasitoide evaluado. Las larvas y parasitoides emergidos se separaron diariamente del recipiente después del conteo.

Los ensayos con cada especie de parasitoides se establecieron con un diseño experimental completamente aleatorizado (DCA), con tres repeticiones y un testigo absoluto sin presencia de parasitoides.

\section{Efecto depredador de Chrysoperla carnea sobre huevos de $C$. viridivittatus}

En la evaluación de C. carnea se empleó la misma metodología que en el ensayo anterior y el mismo tipo de unidad experimental. En cada recipiente plástico se ubicaron los huevos de C. viridivittatus (Tab. 1B) y se introdujo posteriormente una larva de segundo instar del depredador C. carnea, excepto en el testigo.

Las unidades experimentales se observaron diariamente, por un periodo de $15 \mathrm{~d}$, tiempo de duración máxima del estado de huevo de C. viridivittatus, antes de su emergencia. Se tomaron datos como el número de larvas del picudo emergidas y el número de huevos consumidos por C. carnea.

El ensayo se estableció con diseño experimental completamente aleatorizado (DCA), con tres repeticiones y un testigo absoluto sin depredador. En todos los ensayos se realizó análisis de varianza (ANOVA) y Prueba de Comparación de Medias de Tukey (95\%) utilizando el programa estadístico SAS V. 8.1.

\section{RESULTADOS Y DISCUSIÓN}

\section{Potencial parasítico de Trichogramma pretiosum en huevos de $C$. viridivittatus}

En la evaluación del efecto parasítico de $T$. pretiosum sobre huevos de C. viridivittatus en condiciones de laboratorio, el análisis de varianza indicó diferencias significativas $(P \leq 0,05)$ entre los tratamientos evaluados respecto del porcentaje de parasitismo.

La prueba de comparación de medias de Tukey corroboró dichas diferencias, siendo los tratamientos de 45 y 30 individuos de $T$. pretiosum los que brindaron mayor control sobre los huevos del picudo, con porcentajes de parasitismo de 82,5 y 79,3\% (Fig. 2A). Aunque la densidad de 15 individuos presentó el menor control entre los tres tratamientos evaluados, el porcentaje de parasitismo de $72,7 \%$ se considera un valor importante. En el tratamiento control los huevos se desarrollaron de manera normal, permitiendo una emergencia casi completa de las larvas de C. viridivittatus $(92,08 \%)$.

Los resultados obtenidos son similares a los reportados por Basso et al. (1999), quienes en Uruguay, en plantas de vid, evidenciaron un porcentaje de parasitismo producido por adultos de T. pretiosum sobre Bonagota

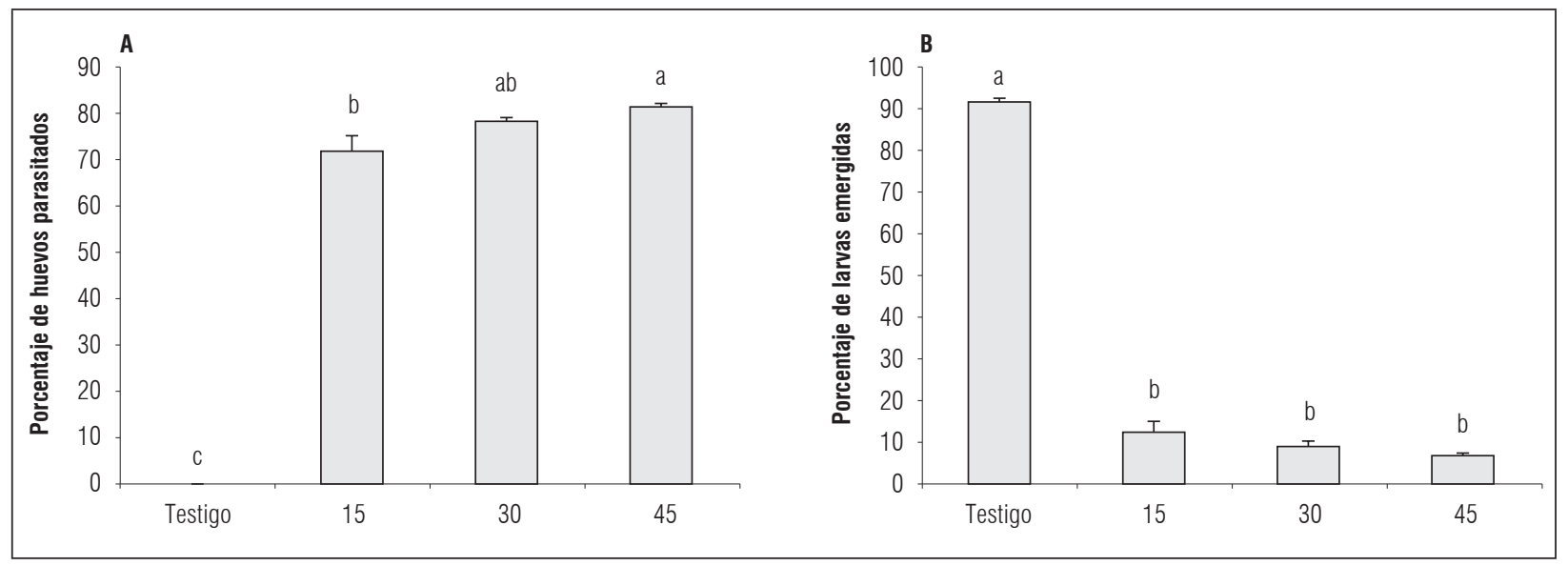

Figura 2. A. Parasitismo y B. Emergencia de larvas en huevos de C. viridivittatus, expuestos a tres densidades de Trichogramma pretiosum en laboratorio. Promedios con letras distintas indican diferencia significativa según la prueba de Tukey $(P \leq 0,05)$. 
cranaodes (Lepidoptera: Tortricidae) del 82,5\%, igual al que se obtuvo en el presente estudio con 45 parasitoides, pero sobre un coleóptero. Por otro lado, Estrada y Soto (2011) con Fidiobia sp. (Hymenoptera: Platygastridae) obtuvieron un parasitismo menor (55\%) al de este estudio, sobre huevos de Compsus sp. en cultivos de cítricos, cuando se liberaron 500 adultos del parasitoide. Lo anterior puede indicar que aunque $T$. pretiosum se recomienda generalmente para el manejo de lepidópteros, es potencialmente útil para el control de C. viridivittatus en vid, toda vez que los huevos son colocados en las hojas.

Respecto al porcentaje de emergencia de larvas de C. viridivittatus y acorde con los resultados anteriores, se observaron diferencias significativas entre los tratamientos y el testigo $(P \leq 0,05)$, obteniéndose valores de emergencia de 12,$5 ; 9,0$ y $6,8 \%$, para 45,30 y 15 individuos de T. pretiosum, respectivamente, lo cual indica que cualquiera de las densidades de parasitoides utilizadas, pueden generar una alta mortalidad de huevos de esta plaga (Fig. 2B, Fig. 5A).

\section{Potencial parasítico de Trichogramma exiguum en huevos de $C$. viridivittatus}

En la evaluación del efecto parasítico de T. exiguum sobre huevos de C. viridivittatus en condiciones de laboratorio, el análisis de varianza indicó diferencias significativas entre los tratamientos $(P \leq 0,05)$, se identificaron tres grupos estadísticamente diferentes (Fig. 3A), siendo el porcentaje de parasitismo, mayor en el tratamiento de 45 parasitoides, con un $69,4 \%$, seguido por 30 y 15 parasitoides con 50,9 y $17,9 \%$, respectivamente.

De acuerdo con lo reportado por Estrada y Soto (2011) para Fidiobia sp. (Hymenoptera: Platygastridae), que presentó un parasitismo de $55 \%$ sobre huevos de Compsus sp. en cultivos de cítricos con 500 adultos del parasitoide, se observa que el valor obtenido en el presente estudio es mayor, en virtud a que empleando menos individuos (45) de T. exiguum se logró cerca del $70 \%$ de parasitismo. Se debe considerar sin embargo, que las condiciones en el presente trabajo fueron controladas en laboratorio, mientras que en el estudio con Fidiobia sp. se realizó con árboles confinados en jaulas en campo.

En T. exiguum se han reportado valores de parasitismo de 80 y 65\% sobre huevos de los tortrícidos Argyrotaenia sp. y Gymnandrosoma aurantianum, respectivamente (Basso et al., 1999), afectando vid en Uruguay; estos difieren en buena parte con los valores aquí obtenidos, posiblemente por las características de especificidad, adaptabilidad biológica y fisiológica de los parasitoides hacia sus hospederos (Madrigal, 2001).

En cuanto a la variable, emergencia de larvas de $C$. viridivittatus, se pudo evidenciar que hubo diferencias significativas entre los tratamientos $(P \leq 0,05)$ estableciendo tres grupos distintos con porcentajes de 74,$0 ; 50,1$ y $18,8 \%$, de emergencia para 15,30 y 45 parasitoides, respectivamente; el tratamiento de 45 parasitoides generó entonces el mayor porcentaje de parasitismo sobre los huevos de C. viridivittatus, afectando la emergencia de larvas del picudo (Fig. 3B, Fig. 5B).


Figura 3. A. Parasitismo y B. Emergencia de larvas en huevos de C. viridivittatus, expuestos a tres densidades de Trichogramma exiguum en laboratorio. Promedios con letras distintas indican diferencia significativa según la prueba de Tukey $(P \leq 0,05)$. 
Lo anterior indicaría que se puede lograr un alto porcentaje de parasitismo de Trichogramma exiguum sobre huevos de C. viridivittatus, pero empleando densidades altas o proporciones mayores a 1:5 (huésped: parasitoide), lo cual indicaría que una estrategia inundativa sería más aconsejable con este parasitoide.

Las variaciones en el porcentaje de parasitismo pueden deberse a varios factores como calidad del hospedero, hábitat, condiciones climáticas, especie de parasitoide (Zago et al., 2007) y cada especie de Trichogramma spp. presenta características distintas en cuanto a capacidad de dispersión y parasitismo del hospedero (Pinto et al., 2003).

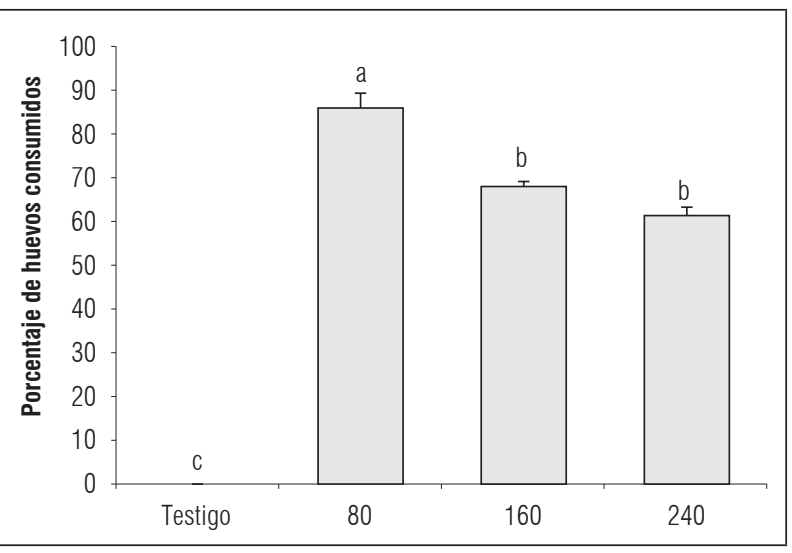

Figura 4. Depredación de huevos de $C$. viridivittatus, por larvas de Chrysoperla carnea sometidas a tres densidades de presa. Promedios con letras distintas indican diferencia significativa según la prueba de Tukey $(P \leq 0,05)$.
En el presente trabajo, la variable emergencia de parasitoides no se pudo cuantificar, en virtud a que luego de ser parasitados los huevos, trascurrieron varios días, observándose cambios en la coloración, pero en ningún momento hubo emergencia de parasitoides; cabe resaltar que los parasitoides con los cuales se realizó la investigación, son multiplicados comercialmente en huevos del lepidóptero Sitotroga cerealella y además las condiciones medio ambientales variaron desde el sitio de producción (Palmira, Valle del Cauca) al sitio del ensayo, lo cual pudo influir en la viabilidad del parasitoide, evitando que cumpla todo su ciclo de vida dentro de los huevos de C. viridivittatus.

Así mismo, diferencias debidas a características intrínsecas del hospedero como ancho del corion, área superficial del huevo (Smith, 1994), calidad nutricional del huevo, presencia de volátiles (Pratissoli et al., 2004) y a la misma cantidad de huevos expuestos (Polanczyk et al., 2011), pudieron alterar el nivel de parasitismo y el normal desarrollo de los parasitoides evaluados.

\section{Efecto depredador de Chrysoperla carnea en huevos de $C$. viridivittatus}

En la evaluación del efecto depredador de Chrysoperla carnea sobre huevos de C. viridivittatus, en laboratorio, no se presentaron diferencias significativas entre las densidades de presa de 160 y 240 huevos, pero si con respecto a la de 80 huevos $(P \leq 0,05)$. En la prueba de Tukey se estableció que hay dos grupos estadísticamente diferentes, en relación con el porcentaje de
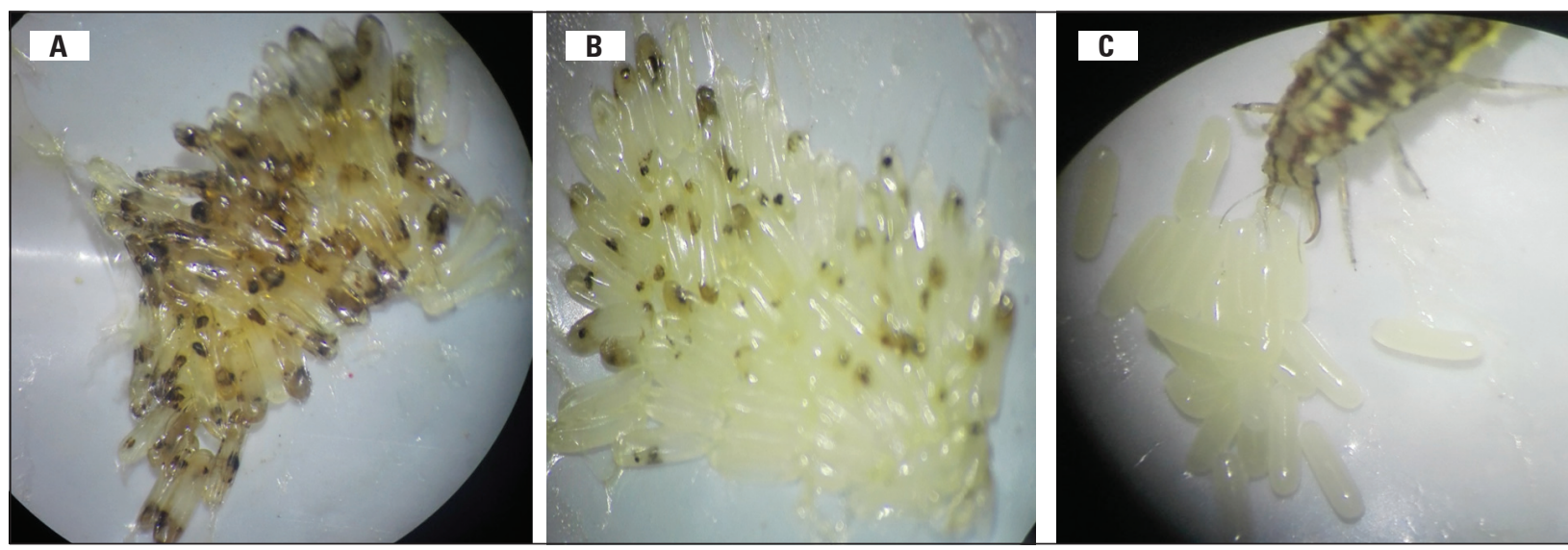

Figura 5. Huevos de C. viridivittatus, parasitados por A. T. pretiosum; B. T. exiguum y C. Consumidos por C. carnea, en condiciones de laboratorio. 
huevos consumidos; el mayor consumo se observó en la densidad de presa de 80 huevos con un $85,8 \%$. En las densidades de 160 y 240 huevos, el porcentaje de huevos consumidos fue menor, pero siempre se ubicó por encima del $50 \%$ (67,9 y $61,2 \%$, respectivamente) (Fig. 4), lo cual indicaría un nivel de consumo potencialmente útil para afectar la población de C. viridivittatus en condiciones de campo.

En la densidad de presa de 80 huevos, como se mencionó, C. carnea ejerció una depredación superior al $80 \%$; estos resultados son similares a los reportados por Castro et al. (2016) en donde se observó un consumo del $80 \%$ de ninfas de $T$. vaporariorum por $C$. externa, con una densidad de presa de 75 individuos. Se resalta que en ambos estudios las condiciones de temperatura y humedad relativa fueron similares, en virtud que se realizó en el mismo laboratorio, aunque con diferente presa.

Según se observa en la figura 4, se evidenció una menor capacidad de consumo de C. carnea a mayor densidad de huevos de C. viridivittatus, caso contrario a lo reportando por Hassanpour et al. (2015) quienes obtuvieron un consumo promedio por parte de $C$. carnea, de 150 individuos, a pesar de emplear diferentes densidades de presa, aunque con individuos de Aphis fabae.

Los anteriores resultados demuestran que probablemente una densidad de presas de 80 huevos de C. viridivittatus, es suficiente alimento para el depredador, como lo sugieren autores como Barbosa et al. (2008), quienes mencionan que una vez se consumen la cantidad de presas necesarias para su desarrollo, las larvas de C. externa reducen su nivel de consumo, evidenciando una respuesta de tipo funcional.

Estudios específicos de depredación de estados inmaduros de Coleópteros como huevos o larvas por Chrysoperla spp. no son muy evidentes en la literatura científica, aunque se mencionan algunos usos en control de Crysomelidos como Leptinotarsa decemlineata en cultivos de berenjenas. Estas aplicaciones se basan en las características principales de este grupo de depredadores como su amplio rango de presas, considerándolas de hábitos polífagos, especialmente las larvas de uso comercial y su eficiencia en la depredación, toda vez que las larvas consumen una gran cantidad de alimento para completar su desarrollo (Pappas et al., 2011).

No obstante, las observaciones realizadas, se debe complementar los estudios de consumo de huevos de
C. viridivittatus por C. carnea, para concluir al respecto, toda vez que el área experimental que se implemente, especialmente si es a pequeña escala, puede afectar el nivel de consumo, en virtud que el depredador encuentra a su presa con mayor rapidez (Montserrat et al. 2000), como se observó en el presente estudio (Fig. 5C). Por lo anterior, sería conveniente desarrollar estos estudios sobre plantas en confinamiento para establecer la capacidad de búsqueda de los huevos de C. viridivittatus por C. externa en condiciones menos controladas.

\section{CONCLUSIONES}

El porcentaje de parasitismo sobre huevos de Compsus viridivittatus, con densidades desde 15 individuos de Trichogramma pretiosum fue superior al $70 \%$; mientras tanto, en el caso de Trichogramma exiguum, densidades inferiores a 45 individuos no representaron un efecto negativo significativo sobre la emergencia de las larvas de C. viridivittatus.

Chrysoperla carnea ejerció una acción depredadora superior al $80 \%$ sobre huevos de C. viridivittatus, cuando existe una proporción 1: 80 (depredador: presa).

Trichogramma pretiosum y Chrysoperla carnea pueden ser consideradas herramientas potenciales de control biológico de C. viridivittatus, en el cultivo de vid en Boyacá, aunque se requieren pruebas de campo para su validación.

\section{AGRADECIMIENTOS}

A la Dirección de Investigaciones DIN, Grupo Manejo Biológico de Cultivos de la Universidad Pedagógica y Tecnológica de Colombia y Viñedo Umaña Dajud, por la financiación del proyecto SGI 1913.

Conflicto de intereses: el manuscrito fue preparado y revisado con la participación de los autores, quienes declaran no tener algún conflicto de interés que coloquen en riesgo la validez de los resultados aquí presentados.

\section{REFERENCIAS BIBLIOGRAFICAS}

Almanza, P., G. Fischer, A. Herrera, A. Jarma y H. Balaguera. 2012. Physicochemical behavior of 'Riesling $\times$ Silvaner' grapevine fruit under the high-altitude conditions 
of Colombia (South America). J. Appl. Bot. Food Qual. 85(1), 49-54. Doi: 10.17584/rcch.2012v6i1.1283

Amalin, D.M., J.E. Peña y R.E. Duncan. 2005. Effects of host age, female parasitoid age, and host plant on parasitism of Ceratogramma etiennei (Hymenoptera: Trichogrammatidae). Fla. Entomol. 88(1), 77-82. Doi: 10.1653/0015-4040(2005)088[0077:EOHAFP]2.0.CO;2

Barbosa, L., C. Carvalho, B. Souza y A. Auad. 2008. Eficiência de Chrysoperla externa (Hagen, 1861) (Neuróptera: Chrysopidae) no controle de Myzus persicae (Sulzer, 1776) (Hemiptera: Aphididae) em pimentáo (Capsicum annum L.). Ciênc. Agrotec. 32(4), 1113-1119. Doi: 10.1590/S1413-70542008000400012

Basso, C., G. Grille y B. Pintureau. 1999. Eficacia de T. exiguum y $T$. pretiosum en el control de Argyrotaenia sphaleropa y Bonagota cranaodes en la vid en Uruguay. Agrociencia 3(1), 20-26.

Cano, D., P. Bustillo, R. Cárdenas y L. Orozco. 2002. Biología y enemigos nativos del picudo de los cítricos Compsus n. sp. (Coleoptera: Curculionidae). Rev. Colomb. Entomol. 28(1), 34-41.

Carrillo, D., J.E. Peña y J.L. Capinera. 2008. Effect of host plants on successful parasitism by Haeckeliania sperata (Hymenoptera: Trichogrammatidae) on Diaprepes abbreviatus (Coleoptera: Curculionidae) eggs. Environ. Entomol. 37(6), 1565-1572. Doi: 10.1603/0046-225X-37.6.1565

Castro, M., J.W. Martínez y M. Dotor. 2016. Evaluación del efecto regulador de Chrysoperla externa sobre mosca blanca Trialeurodes vaporariorum en tomate. Rev. Cienc. Agr. 33(2), 43-54. Doi: 10.22267/rcia.163302.51

Cerna, A., J. Landeros, S. Sánchez, M. Badii, L. Aguirre e Y. Ochoa. 2012. Comparación de la toxicidad y selectividad de insecticidas para la plaga Bactericera cockerelli y su depredador Chrysoperla carnea. Agrociencia 46, 783-793.

Estrada, G. y A. Soto. 2011. Parasitismo de Fidiobia sp. (Himenóptera: Platygastridae) sobre Compsus sp. (Coleóptera: Curculionidae). Bol. Cient. Mus. Hist. Nat. Univ. Caldas 15(2), 201-205.

Garcia, F., R. Mercado, A. González y M. Ramírez. 2011. Especies nativas de Trichogramma (Hymenoptera: trichogrammatidae) colectadas en cultivos agrícolas del norte de México. Rev. Chapingo Ser. Cienc. For. Ambient. 17(ed. esp.), 173-181. Doi: 10.5154/r. rchscfa.2010.09.070

Hassanpour, M., R. Maghami, H. Rafiee-Dastjerdi, A. Golizadeh, M. Yazdanian y A. Enkegaard. 2015. Predation activity of Chrysoperla carnea (Neuroptera: Chrysopidae) upon Aphis fabae (Hemiptera: Aphididae): effect of different hunger levels. J. Asia Pac. Entomol. 18, 297-302. Doi: 10.1016/j.aspen.2015.03.005

Madrigal, A. 2001. Generalidades sobre himenóptera parasitoides. pp. 83-120. En: Fundamentos de control biológico de plagas. Universidad Nacional de Colombia, Medellín, Colombia.
Marín, P. y A. Bustillo. 2008. Selección de aislamientos de Beauveria bassiana virulentos a Compsus n.sp. Cenicafe 59(2), 165-173.

Montserrat, M., R. Albajes y C. Castane. 2000. Functional response of four heteropteran predators preying on greenhouse whitefly (Homoptera: Aleyrodidae) and Western flower thrips (Thysanoptera: Thripidae). Environ. Entomol. 29, 1075-1082. Doi: 10.1603/0046-225X-29.5.1075

Nasreen, A., G. Mustafa y M. Ashfaq. 2005. Mortality of Chrysoperla carnea (Stephens) (Neuroptera: Chrysopidae) after exposure to some insecticides; laboratory studies. S. Pac. Stud. 26(1), 1-6.

Navarro, M.A. 1990. Producción, uso y manejo en Colombia: el Trichogramma spp. ICA, Palmira, Colombia.

O’Brien, C.W. y J. Peña. 2012. Two species of Compsus Schoenherr, new citrus pest from Colombia (Coleoptera: Curculionidae: Entiminae). Insecta Mundi 0227, $1-13$.

Oliveira, H., J. Zanuncio, D. Pratisssoli e I. Cruz. 2000. Parasitism rate and viability of Trichogramma maxacalii (Hym.: Trichogrammatidae) parasitoid of the eucalyptus defoliator Euselasia apisaon (Lep.: Riodinidae), on eggs of Anagasta kuehniella (Lep.: Pyralidae). For. Ecol. Manage. 130, 1-6. Doi: 10.1016/ S0378-1127(99)00172-3

Pappas, M.L., G.D. Broufas y D.S. Koveos. 2011. Chrysopid predators and their role in biological control. J. Entomol. 8(3), 301-323. Doi: 10.3923/je.2011.301.326

Peñaloza, C. y R. Diaz. 2004. Así se maneja y controla el picudo de los cítricos Compsus sp. ICA, Bogotá, Colombia.

Pinto, A., J. Parra, H. Oliveira y E. Arrigoni. 2003. Comparação de técnicas de liberação de Trichogramma galloi Zucchi (Hymenoptera: Trichogrammatidae) para o controle de Diatraea saccharalis (Fabricius) (Lepidoptera: Crambidae). Neotrop. Entomol. 32, 311-318. Doi: 10.1590/S1519-566X2003000200018

Polanczyk, R., W. Barbosa, F. Celestino, D. Pratissoli, A. Holtz, A. Milanez, J. Cocheto y A. Silva. 2011. Influência da densidade de ovos de Diaphania hyalinata (L.) (Lepidoptera: Crambidae) na capacidade de parasitismo de Trichogramma exiguum Pinto \& Platner e Trichogramma pretiosum Riley (Hymenoptera: Trichogrammatidae). Neotrop. Entomol. 40(2), 238-243. Doi: 10.1590/S1519-566X2011000200013

Pratissoli, D., A. Holtz, J. Gonçalves y R. De Oliveira. 2004. Características biológicas de linhagens de Trichogramma pretiosum, criados em ovos de Sitotroga cerealella e Anagasta kuehniella. Hortic. Bras. 22, 562-565. Doi: 10.1590/S0102-05362004000300012

Rincón, E. y C. Montoya. 2015. Evaluación de la eficacia de Metarhizium anisopliae y Beauveria bassiana en el control de picudos asociados con la vid, utilizando activadores de patogenicidad. Novedades Técnicas 24, 5-12. 
Smith, S. 1994. Methods and timing of releases of Trichogramma to control lepidopterous pests. p. 113-144. En: Wajnberg, E. y S.A. Hassan (eds.). Biological control with egg parasitoids. CABI Publishing, Wallingford, UK.

Van Driesche, R., M. Hoddle y T. Center. 2007. Control de plagas y malezas por enemigos naturales. U.S. Department of Agriculture (USDA), Washington DC, USA.

Van Huis, A., M.G. Wijkamp, P.M. Lammers, C.G.M. Klein Goldewijk, J.H. van Seeters y N.K. Kaashoek. 1991. Uscana lariophaga (Hymenoptera: Trichogrammatidae), an egg parasitoid of bruchid beetle (Coleoptera: Bruchidae) storage pests in West Africa: host-age and host-species selection Bull. Entomol. Res. 81(1), 65-76. Doi: $10.1017 /$ S0007485300053256
Yadav, R. y P. Pathak. 2010. Effect of temperature on the consumption capacity of Chrysoperla carnea (Stephens) (Neuroptera: Chrysopidae) reared on four aphid species. Bioescan 5(2), 271-274.

Zago, H., D. Pratissoli, R. Barros, J. Gondim y J. Dos Santos. 2007. Capacidade de parasitismo de Trichogramma pratissolii Querino \& Zucchi (Hymenoptera: Trichogrammatidae) em hospedeiros alternativos, sob diferentes temperaturas. Neotrop. Entomol. 36(1), 84-89. Doi: 10.1590/S1519-566X2007000100010

Zuluaga, P.A., A.M. Caicedo, N. Cardozo, J.E. Muñoz y A. Carabalí. 2015. Entomopatogenos asociados a citricos y su patogenisidad sobre Compsus viridivittatus Guérin-Méneville (Coleoptera: Curculionidae: Entiminae). Corpoica Cienc. Tecnol. Agropecu. 16(2), 293305. Doi: 10.21930/rcta.vol16_num2_art:374 